\title{
A Hybrid Emotional Facial Expression Recognition Algorithm using Enhanced Gabor Filter with AdaBoost Classifier
}

\author{
${ }^{1}$ Shaik Taj Mahaboob, ${ }^{2} \mathrm{~S}$. Narayana Reddy and ${ }^{3} \mathrm{P}$. Jagadamba \\ ${ }^{1}$ Department of ECE, JNTUA College of Engineering Pulivendula, Pulivendula, India \\ ${ }^{2}$ Department of ECE, SVU College of Engineering, Tirupathi, India \\ ${ }^{3}$ Department of ECE, Srikalahasteeswara Institute of Technology, Srikalahasti, India
}

\begin{abstract}
Facial expression recognition is considered to be the major research area in the context of pattern recognition and image processing is considered as a vibrant research problem. The study presented in this study, demonstrates a novel algorithm developed based on the integration of Gabor filter and DCT (Discrete Cosine Transform) along with AdaBoost classifier to enhance the recognition rate. Experimental results show that the proposed technique outperforms diverse existing mechanisms in terms of recognition rate while implemented on various standard facial image databases.
\end{abstract}

$\underline{\text { Key words: Face expression recognition, Gabor filter, DCT, AdaBoost, emotion recognition, demonstrates }}$

\section{INTRODUCTION}

From the last few decades face recognition became popular which provides security to the data or something which is more important for now and further use. Because of this increasing demand, number of techniques came into existence even though it is still a challenging problem (Ramasubramanian and Venkatesh, 2001) because of its complexities and variations in image poses, expression and soon. There are many techniques to recognize the face, these techniques are classified into three types, i.e., generative, holistic and feature or pose based recognition methods. The generative method is used to assume the observation features of an image (Agrwal et al., 2016). The holistic method will use global information from faces which can be represented by number of features and these features will capture the number of variations of independent faces. To recognize the face in holistic approach we have two methods such as Karhunen-Loeve expansion and linear discriminant analysis (Shan et al., 2009). The another method is feature or pose based method which is used to extract the features like nose, eyes and soon and poses like expressions, etc. These feature based method is again divide into two types, i.e., geometric and non-geometric approaches. In geometric approach, only from the particular area the features of an image are extracted, i.e., nose, eyes, etc. In the non-geometric approach, the expressions are extracted from face to recognize the original image. The expressions are the effective way to communicate with the people. It has great impact on the communication without expression we cannot predict the mood of a human. If, we detect the pose of a human, then, we have two approaches, i.e., multi view face recognition which compare the image with other images of same situation in a gallery and probe faces which is used to compare with the several images in a gallery.

Secondly, to detect the expression of a human we have to follow five steps such as image acquisition which is used to extract the image from a complex environment. Preprocessing is used to track the pats of a face clearly (Gupta et al., 2011). Feature extraction which is used to classify the image based on expressions, poses and motions, etc. In classification approach, the image is classified into parametric or non-parametric based on the expressions of an image. Finally, the post processing approach is used to correct the errors by improving the accuracy and delivers an effective and efficient image. The image features can be extracted mainly by using the following techniques such as scale-invariant feature extraction, Local Binary Pattern (LBP), histogram of gradient, Gabor filter, Discrete Wavelet Transform (DWT) Discrete Cosine Transform (DCT). Among all these techniques the mainly used techniques are DWT, DCT and Gabor filters. By using DWT we easily transform the image into coefficients of frequencies, the low frequencies have essential and unique features of an image. To extract the edge patterns of an image, we use zig-zag approach of discrete cosine transforms. While extracting the features some data redundancy has occurred to reduce the redundancies we have to use some dimensionality methods such as PCA, ICA and LDA (Tariq and Huang,

Corresponding Author: Shaik Taj Mahaboob, Department of ECE, JNTUA College of Engineering Pulivendula, Pulivendula, India 
2012). Other researchers proposed some techniques such as discrimination power analysis technique, illumination normalization technique and random transform, etc. After reducing the redundancies, the Gabor filter is used to enhance the performance of face recognition method which have different combinations of distribution. But it is robust for its assumptions and changes of facial recognition.

This research study mainly concentrates on developing a hybrid technique that includes Gabor and DCT such that initially the features extracted using Gabor filter are reduced to optimum using the DCT reduction technique, further the recognized emotions are classified using advanced AdaBoost classifier.

Literature review: The face recognition technique is used in many applications but in some applications such as criminal cases are for security purpose a gesture of face also has to be stored. So, for this reason several approaches were suggested to derive new techniques for face gesture recognition is Gabor-Max-DCT (Verma et al., 2013) feature extraction techniques. Face gesture recognition is a part of pattern recognition. The Gabor filter will found the edge pattern values and texture of an image with high dimensions and high redundant values. Then, it forms a matrix with the obtained redundant values to reduce the redundancies in an image. The matrix has some redundancies to reduce those redundancies researchers proposed a two level reduction technique. In the first level, a low frequency value has to be selected to get the effective facial gestures. In the next level, discrete cosine transform is used to reduce the remaining redundancies of an image. In discrete cosine transform, a zig-zag method is used which is same as low frequency method to reduce the redundancies and it produced an effective and accurate face gesture recognition. This algorithm mainly needs MATLAB to execute. In this study, the researcher also compares the proposed technique with other techniques to prove that the proposed technique gives high accuracy face gesture recognition. It gives $90 \%$ accurate results when compared with the local binary pattern. In future, we also enhance the proposed algorithm to detect the face in video.

To recognize faces by using 2 and 3 dimensional intensity images independently has some problems in reliability and clarity. So, in an study (Tariq and Huang, 2012) a technique by using Gabor discrete cosine transform called 2.5 dimensional face recognition technique. Here, we preprocess the image to find the region of interest in a face of image and also it removes noises from the image. It produces clear and clean data to extract the face from an image and also it finds the depth and texture of an image for easy face recognition. After preprocessing technique a image has to be filtered by using Gabor filter. According to the Gabor filter, the image is divided into two parts, i.e., amplitude and phase. For face recognition it uses amplitude which contains 40 magnitude coefficients and all these coefficients can be drawn out from the input image. By these coefficients Gabor image can be obtained. Discrete cosine transform will fragment the image into frequency components, i.e., it transforms the image into frequency to find the redundancies and also to reduce the dimensions of an image (Shan et al., 2009). It also encodes the finite details of image to differentiate it from the other facial details. After encoding, the frequencies of an image, a classifier such as $k$-nearest neighbors is used to classify all the nearest frequencies into a group. The combination of all these frequencies can form an encoded image, finally, the image is decoded and the face is recognized accurately. Comparing 2D and 3D independently don't work effectively with the combination $2 \mathrm{D}$ and $3 \mathrm{D}$ that is $2.5 \mathrm{D}$. The 2.5D technique gives best and high performance, increased accuracy and reliability (Ou et al., 2010).

Bhadu et al. (2012) proposed some techniques such as Discrete Cosine Transforms (DCT), Gabor and wavelet feature extraction for facial expression recognition. The facial expression recognition plays a very important role in any type of communication, i.e., either verbal or non-verbal. This is part of pattern recognition and image processing. To recognize the facial expression first we have follow 3 phases, i.e., facial detection, facial extraction and classifier. After detecting the face in an image, the expressions have to be extracted from the image by using discrete cosine transforms, Gabor filters and wavelets. In DCT, the image can be extracted by reducing the redundancies using zig-zag technique. In low frequency, the outline features of a image can be specified and the noise also eliminated from the image. The advantages of DCT are independent, providing better results in low frequency and energy usage is also low. The main disadvantages are it is sensitive to change and coefficients magnitude is invariant. Other image extraction technique is Gabor filter, it is used to extract the edge patterns of an image with different frequencies and construct a matrix for the Gabor frequencies to reduce the redundancies and removes the noise from the image to produce clean clear image. In this Gabor filter, it mainly checks the coefficients of important features of face, i.e., nose, eyes, mouth and ears, etc. it mainly focuses on some features such as transformation, rotation and scale and these features are constant because of these advantages the Gabor filter can extract the clean image. The main disadvantage of Gabor filter is it defines high dimensions of an image, so, it takes time to extract the 
image and it doesn't provide exact features of face in an image. With the usage of maximal spatial localization, the Gabor filter is not optimal. The other type of image extraction is wavelet transform, it decomposes the image into several levels and each level has some filters. In the first level, it uses one pass filter to extract the image features and in other levels the extracted image is taken as input and perform the two pass filter to extract the clear image from the 2D or 3D image (Lajevardi and Lech, 2008). The main advantage of wavelet transform is to extract the essential features and frequencies from the image. After extracting the image features, we have to classify them using some classifiers such as voting algorithm. With this classifier algorithm the image can be extracted in an efficient and with accurate way. In this study, we compare the image extraction techniques with each other to know effective extraction technique. Among all the above techniques, the discrete cosine transform is efficient with the others. In future, we can use other new extraction techniques for getting an effective way to recognize the face.

Tyagi and Khanna (2012) suggested other technique for face recognition is the combination of discrete cosine transform and nearest neighbor discriminant analysis. In DCT, the whole image is taken as input and then, the essential features are drawn out which contains low and high frequency coefficients. Among all coefficient values select few low frequency coefficients and represent matrix of same dimensions. These coefficients are represented in the form of matrix and which can be used for future scope. This matrix can be taken as input to the discrimination analysis. This discrimination analysis can be performed by using nearest neighbor discriminate analysis here, we use two-level structure for noisy image. To remove this noisy image and to make them as smoothed image, we have to zeroing the vertical coefficients of image of DWT. Here, we use training algorithm, i.e., combination of DCT and NNDA which classifies the data into classes and samples and it forms matrix for the obtained classes and samples. Finally, we get a clear image from the obtained matrix. Now, we have compare the obtained image whether it is correct or not by using Euclidian distance. When the two images have the same distance between the coefficients, then we can say that the same image is extracted from the original image.

\section{MATERIALS AND METHODS}

Proposed technique: Facial expression recognition is considered as most prominent pattern recognition technique at the application level which is playing vital role now a days in the context of solving criminal cases as well as deep investigation of any situation. Even though

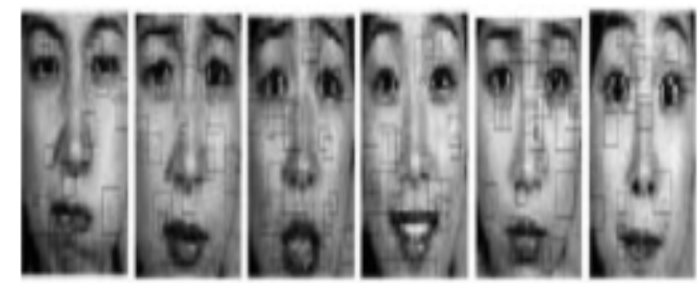

Fig. 1: Sample set of various expressions

several research studies addressed various challenges in the concept of facial expression recognition, till date, there are many open issues that includes illumination, occlusion and accuracy that are needed to be optimized. These issues can be normalized if and only if an absolute feature extraction patterns are analyzed. The unique features that are to be extracted in a facial gesture is its texture and shape.

In general, the concept facial feature extraction is classified as non-geometric and geometric approaches. In geometric patterns, the features are initially extracted from the particular geometric segment of the face while in the case of non-geometric facial expression extraction is the complete face is considered the extraction process is carried out with the Gabor filter and local binary patterns. Several research studies stated that DCT (Discrete Cosine Transformation) is the most efficient technique to retrieve facial features effectively in the context of the shape and texture along with the minimized frequency coefficients.

The process of Gabor filter feature extraction is constructed on the prognosis of Gaboron facial images with variability of scale and projectionangle. Gabor feature, extraction is considered to be robust in nature due to the scale, illumination and expression changes in the context of the facial emotion recognition applications. Geometric based emotion extraction approaches initially concentrate on particular region of facial aspects like eyes and mouth such that these facial regions are segmented such that the low dimensional features are extracted. State-of-art mechanisms include the hybrid techniques for feature extraction such that, few features are extracted from the local region of the face and further are extracted from the global aspect of the face.

The conceptual representation of the proposed method is depicted in Fig. 1. The proposed emotion recognition algorithm consists of five steps, the initial step focus on the preprocessing to identify the texture data and depth data of the face further Gabor filter is applied to retrieve the features of emotion and further a hybrid DCNNDA-algorithm is used to identify the facial emotions, finally, an Ada-Booster classifier is used to classify the identified expressions (Fig. 2). 


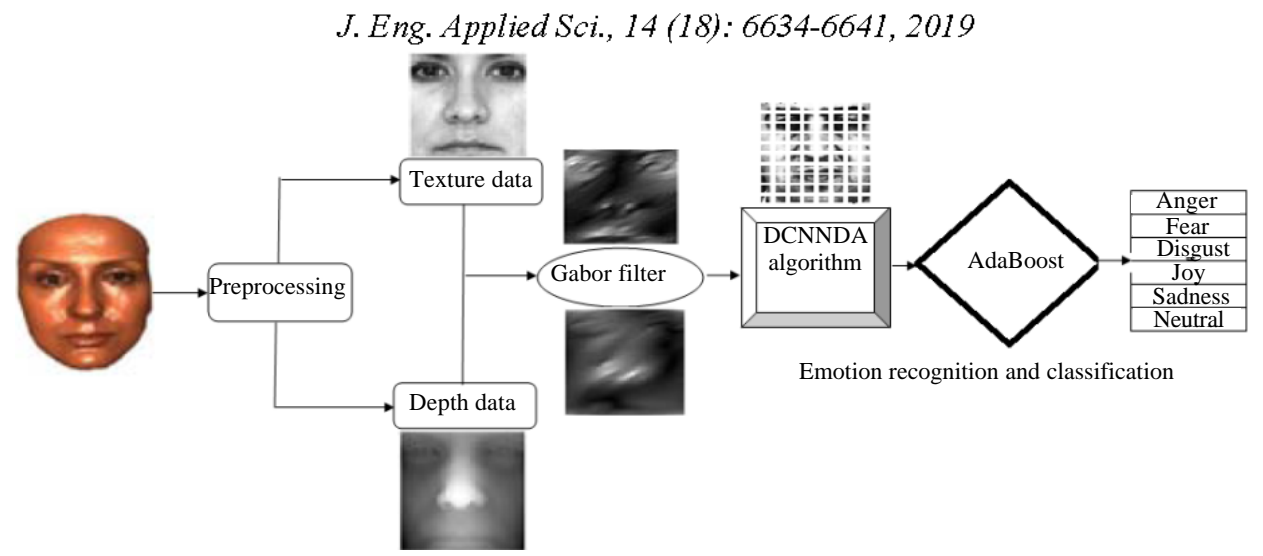

Fig. 2: Proposed method

Preprocessing: In the context of analyzing multidimensional faces, the faces acquired from the dataset may consists of noise where the geometric coordinates of the interested facial regions are not appropriate. Hence, preprocessing of this dataset is highly required before the feature extraction phase. Preprocessing phase is composed in 3 different stages. In the initial stage, the faces obtained from the dataset are ranged to fixed coordinates. In order to restrict the phases to the fixed coordinates, Iterative Closest Point algorithm (Bhadu et al., 2012) is applied. The functional aspects of this algorithm identify the frontal region of the face with neutral emotion and notate it as a fixed aspect. Further, the interested regions of each face is identified such that, eliminating the hair neck and shoulder part and by concentrating of the facial features. To made this aspect possible a $120 \times 120$ rectangular pane regions are utilized. In the final stage a, $3 \times 3$ pane is used to eliminate the features like spike nose. Finally, the depth and texture images are obtained from the given multidimensional image data.

Gabor filter: Feature extraction from the texture and the depth data is processed with the help of the Gabor wavelet. These are defined in the state of art studies (Lajevardi and Lech, 2008) and were extended in to multidimensional space (Tyagi and Khanna, 2012). Initially, a Gabor wavelet is considered to be as multifaceted sinusoids that are modulated from the complex Gaussian function which depicts the properties of spatial frequency, optimal selectivity as well as spatial localization. Gabor image is the convoluted image represented by Gabor kernel and defined as:

$$
\varphi(E, R, \theta, \beta)=\frac{1}{2 \pi \rho E \rho R} e^{\frac{1}{2}\left(\frac{R_{1}^{2}}{\rho_{R}^{2}}+\frac{E_{1}^{2}}{\rho_{\mathrm{E}}^{2}}\right)}
$$

$\mathrm{E}_{1}$ as well as $\mathrm{R}_{1}$ are computed using the projection angle, $\theta$ as represented in the following Eq. 2 :

$$
\left\{\begin{array}{l}
E_{1}=E \cos \theta+R \sin \theta \\
R_{1}=-E \cos \theta+R \sin \theta
\end{array}\right.
$$

In this milieu (E, R), the Gabor Kernel, location is considered to be in multidimensional space. $\beta$ is considered as a wavelength to determine the sinusoidal wavelet phase angle. $\theta$ is evaluated as the projection angle with respect to the Gabor kernel. $\rho x$, pyare computed as the standard deviation from the mean of the direction coefficients generated by $\mathrm{E}$ and $\mathrm{R}$. Equation 3 shows the convolution operation carried out by the Gabor kernel that identify the edge features of the facial emotion:

$$
\mathrm{G}_{\mathrm{n}, \mathrm{m}}=(\mathrm{e}, \mathrm{R})=\mathrm{I}(\mathrm{E}, \mathrm{R})^{*} \varphi(\mathrm{E}, \mathrm{R})
$$

$\varphi(\mathrm{E}, \mathrm{R}, \theta \mathrm{u}, \beta v)$ is defined to be a Gabor kernel is considered to be as a complex number, hence, on utilizing the real part and the convoluted imaginary part, the projection angle is computed, further the joint amplitude is evaluated. As the matrices generated by the Gabor emotion extraction have high redundant features with multidimensional complexity, Gabor computes a multidimensional feature matrix for every orientation such that it includes 5 diverse angles as shown in Fig. 3.

DCT (Discrete Cosine Transform): Signal analysis and image processing prominently uses DCT for its energy compaction property. The vital feature of DCT is that it wraps up the signal based information in to variant of geometric coefficients. In this context, DCT is considered for feature extraction. In the initial cases, DCT is applied on the whole image for dimension reduction that results in the high and low frequency geometric coefficients through matrix such that using zig-zag method, the low frequency vectors such that remaining are discarded. DCT is computed as follows (Fig. 4). 


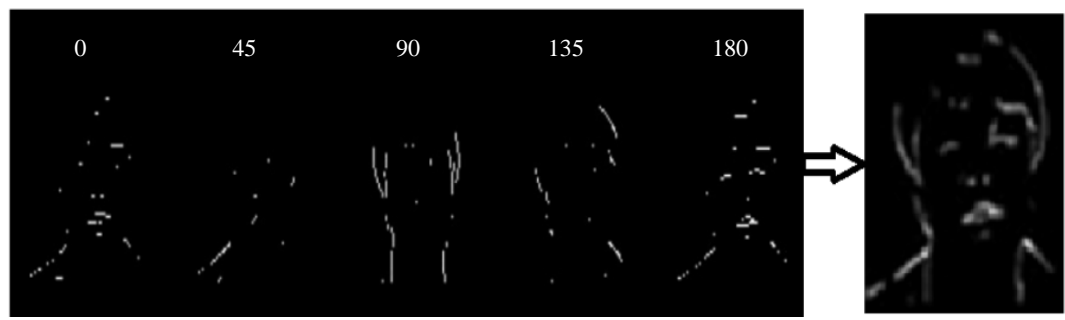

Fig. 3: Gabor feature extraction

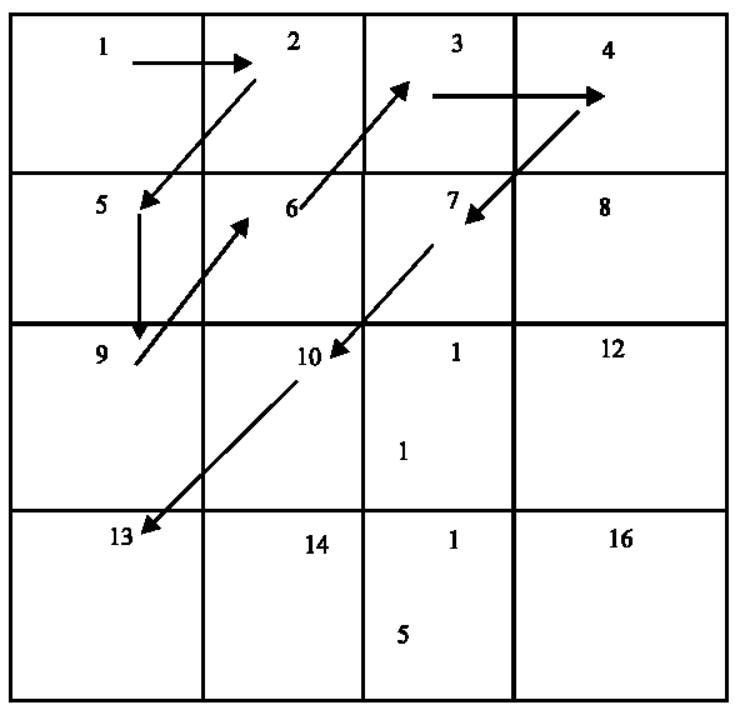

Fig. 4: Zig-Zag feature extraction

Let us assume the facial image as a matrix of $M(k, 1)$ of dimensions $\mathrm{a} \times \mathrm{b}$, then, the discrete transformation value is $\mathrm{m}(\mathrm{o}, \mathrm{p})$ having the dimensionality features as $\mathrm{a} \times \mathrm{b}$ :

$$
\begin{gathered}
\mathrm{m}(\mathrm{o}, \mathrm{p}) \frac{1}{\mathrm{ab}} \alpha(\mathrm{o}) \alpha(\mathrm{p}) \times \sum_{\mathrm{k}=0}^{\alpha-1} \sum_{1=0}^{\mathrm{b}-1} \mathrm{M}(\mathrm{k}, 1) \times \\
\cos \left(\frac{(2 \mathrm{k}+1) \mathrm{o} \pi}{2 \mathrm{a}}\right) \cos \left(\frac{(21+1) \mathrm{p} \pi}{2 \mathrm{~b}}\right)
\end{gathered}
$$

In this context, $\mathrm{k}$ and $\mathrm{l}$ are the geometric coordinates in the specific domain such that o and $p$ are evaluated as the frequency coordinate in the translated domain. Further, the DCT can be integrated with the Nearest Neighbor Discriminant Analysis (NNDA) such that the facial emotions are analyzed accurately.

DCNNDA-algorithm for facial feature extraction: Initially during the process, DCT is applied completely to extract features based the low level frequency selection using zig-zag method is shown in Fig. 3. The algorithm that integrates DCT and NNDA is generated below. Here, let us consider, we have $\mathrm{X}$ classes as well as $\mathrm{Y}$ samples per classes that could derive $\mathrm{X}^{*} \mathrm{Y}$ samples in the training data set.

\section{DCNNDA-algorithm}

1. For every image of size $X^{*} Y$ compute the coefficient matrix

2. Implement Zig-Zag method to extract the geometrical coefficients with lower frequency rates

3. Enhance the extracted facial features in the form of a test bed dataset in to a matrix $\mathrm{X}$

4. To learn NNDA projection matrix initially apply NNDA on $\mathrm{X}$ :

$$
\mathrm{R}_{\mathrm{MNDA}}=\left[€_{1}\left|€_{2}\right| €_{3}|, \ldots,| €_{\mathrm{d}}\right], \mathrm{R}_{\mathrm{MNDA}} \in \mathrm{D}^{\mathrm{d}^{*} \mathrm{Y}}
$$

5. Finally, the projection of the feature matrix is obtained $I=R_{\text {nnda }} A$

Facial expression classification based AdaBoost: Despite of various facial expressions the variety of expressions are related to diverse ranges of motions at each pattern. AdaBoost classifier acts as a two-class classifier that overlays particular weights on a variety of set of weak classifiers that helps in generating a strong classifier. The comprehensive steps of AdaBoost algorithm are depicted as follows.

AdaBoost classifier for expression recognition: Let us assume a sample space $\mathrm{X}$ as well as the set of labeled classes $\mathrm{LC}=\{-1,1\}$ and the training set includes $\left\{\left(\mathrm{X}_{1}\right.\right.$, $\left.\left.\mathrm{LC}_{1}\right),\left(\mathrm{X}_{2}, \mathrm{LC}_{2}\right) \ldots\left(\mathrm{X}_{\mathrm{n}}, \mathrm{LC}_{\mathrm{n}}\right)\right\}$ where $\mathrm{X}_{\mathrm{i}} \in \mathrm{X}$ and $\mathrm{LC}_{\mathrm{i}} \in \mathrm{LC}$ is considered to be the class label.

Initialize the training weights such that every sample weight will have the similar weight $\mathrm{Wt}_{\mathrm{i}}=1 / \mathrm{M}$ where $\mathrm{M}$ indicates the samples count, while training the each sample the process is accumulated to train a feeble classifier such that $£^{(t)}=\mathrm{X} \rightarrow \mathrm{LC}$ and hence, the error $\mathrm{e}^{(\mathrm{t})}$ is mitigated $€^{(t)}=\sum_{i=1}^{M} W t \mid E^{(t)}\left(X_{i}\right)-L_{i}$ and the weight of classifier is:

$$
\mathrm{Wt}_{\mathrm{i}}^{(\mathrm{t}+1)}=\frac{\mathrm{wt} \exp \left(-\alpha € \operatorname{LCif}\left(\mathrm{X}_{\mathrm{i}}\right)\right)}{\sum_{\mathrm{i}=1}^{\mathrm{m}} \mathrm{wt}}
$$

Generation of a strengthened classifier model:

$$
F\left(X_{i}\right)=\left[\sum_{t=1}^{t} \alpha t f(t)\left(X_{i}\right)\right]
$$

\section{RESULTS AND DISCUSSION}

For the purpose of evaluation, multiclass AdaBoost classifier is adopted for classification of facial gestures of 
Table 1: Comparative analysis of recognition rate of various existing mechanisms along with proposed approach on JAFFE database

\begin{tabular}{lc}
\hline Algorithm for feature extraction & Recognition rate (\%) \\
\hline Gabor+SVM+AdaBoost (Ramadan and Abdel-Kader, 2009) & 92.2 \\
PHOG+Gabor+AdaBoost (Tyagi and Khanna, 2012) & 93.1 \\
LBP+Template matching (Abbas et al., 2008) & 78.1 \\
DCT-based feature selection for facial gesture recognition (Liu et al., 2009) & 55.22 \\
DWT-based facial gesture recognition (Chen and Zhang, 2007) & 78.1 \\
Boosted LBP (Sellahewa and Jassim, 2008) & 80.1 \\
Proposed algorithm (Gabor+DCNNDA) & 96.5 \\
\hline
\end{tabular}

Table 2: Comparative Analysis of recognition rate of various existing mechanisms along with proposed approach on CK database

\begin{tabular}{lc}
\hline Algorithm for feature extraction & $\begin{array}{c}\text { Recognition } \\
\text { rate }(\%)\end{array}$ \\
\hline Gabor+SVM+AdaBoost & 89.2 \\
PHOG+Gabor+AdaBoost & 88.1 \\
LBP+Template matching & 81.1 \\
DCT-based feature selection for facial gesture recognition & 65.2 \\
DWT-based facial gesture recognition & 81.1 \\
Boosted LBP & 72.1 \\
Proposed algorithm (Gabor+DCNNDA) & 94.5 \\
\hline
\end{tabular}

JAFFE dataset and CK dataset that consists of a variety of facial gestures and the optimal recognition is evaluated through a cross validation phenomenon where a set of 1 random iteration with respect the set of seven classes of expressions that include \{anger, fear, neutral, sad, happy, surprise, disgust $\}$ such that the dataset consists of 230 grey scale images and the experimental results are tabulated as follows Table 1.

Experiments on JAFFE dataset: In context of implementation the JAFFE database consists of 230 grayscale images out of which 10 are the Japanese female facial expressions. Such that the classification is initiated by identifying the individual facial expression categorization. To demonstrate the proposed model, the concept is further categorizing the facial expressions with the variant of two sub classes that includes either non-smiling or smiling. The mentioned Table 1 evaluate the comparative analysis of various technique along with the proposed hybrid technique over the JAFFE dataset.

Experiments on CK dataset: Robustness of the proposed method is further being evaluated with CK dataset that is considered to be the most comprehensive dataset in the context of facial recognition dataset, initially, this dataset consists of facial images of the student of 100 universities of which the age of the students was in the range of 19-32. The recognition rate of the proposed method is evaluated through CK dataset and the results are tabulated in Table 2 . The observations from Table 2 are summarized as follows, it is found that the proposed method outperforms

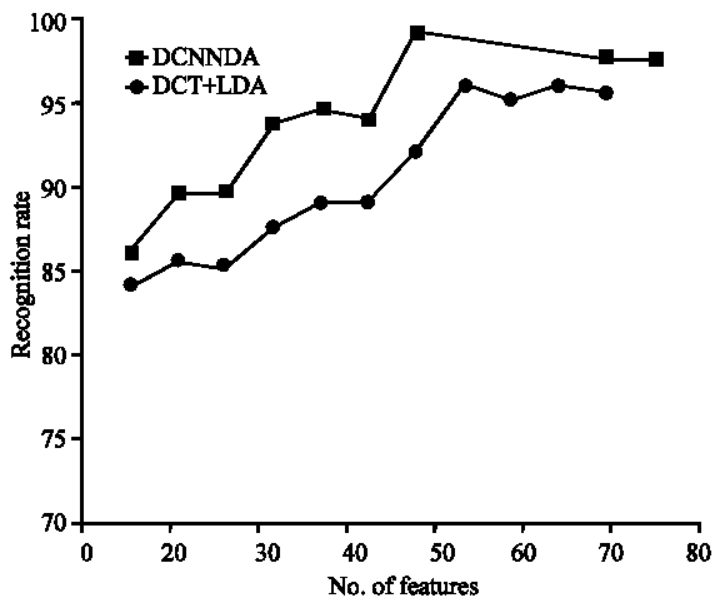

Fig. 5: Performance analysis of DCNNDA on ORL dataset

the various state of art techniques, in terms of the recognition rate. It is also observed that the rate of recognition in the previous algorithms are slightly low when compared with JAFFE dataset recognition accuracy. Further, the reason is analyzed such that the image quality is low in the context of CK dataset where the features in the facial expression are in the lower derivative region.

Furthermore, to analyze the performance of proposed technique with the various dataset like ORL database that literally consists of 450 facial images related to 35 subjects such that it includes around 12 samples per subject with a disparity in the expression and pose. Initially we kept 7 images per subject to develop a training dataset. After implementing the proposed technique in the context of non-matched faces, around $99 \%$ recognition rate at 50 features, Fig. 5 shows performance on diverse number of features. Adding to that the evaluation of the technique is carried out on the Yale dataset (Anonymous, 2019) that consists of around 170 facial image dataset of around 20 people. Similarly, as the above context around 7 images for every class to develop a training dataset. Finally, on the application of the proposed technique $97.3 \%$ of recognition rate as show in Fig. 6. 


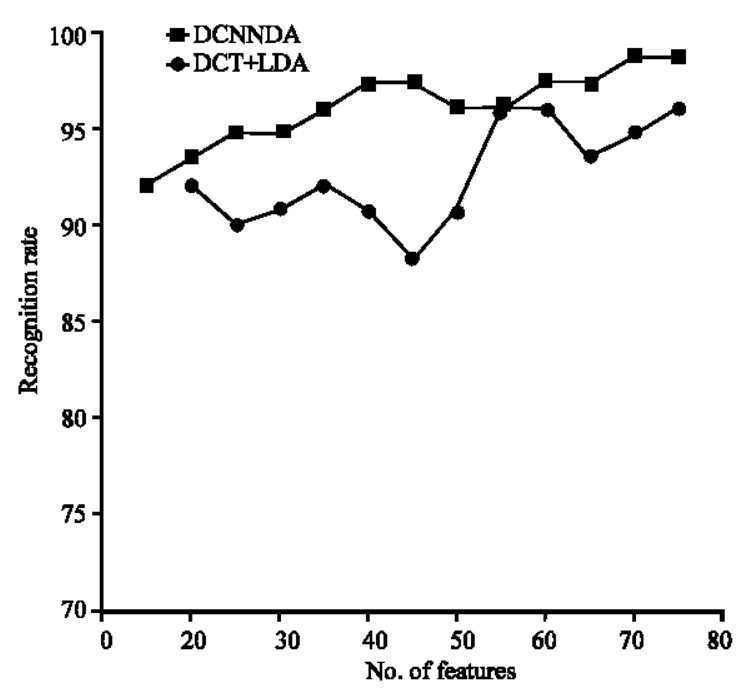

Fig. 6: Performance analysis of DCNNDA on Yale dataset

\section{CONCLUSION}

The main objective of this research is to develop a novel algorithm that even recognize the expressions with in a multidimensional facial dataset with an absolute accuracy. Gabor+DCNNDA algorithm proposed in the research is a hybrid and novel algorithm that was combined with the AdaBoost classifier to recognize the expression. After systematic investigation of the proposed over 4 different facial image datasets that include JAFFE, CK, Yale and ORL, it is observed that the proposed technique outperforms the state-of-art techniques in the context of the recognition rate. Further, the research could be extended to improve the accuracy rate recognizing facial expression of the blur images.

\section{ACKNOWLEDGEMENTS}

We are thankful to Department of ECE, SVU College of Engineering, Sri Venkateswara University, Tirupathi, and JNTUA College of Engineering for providing us opportunity to carry on our research. We are thankfull to our colleagues at SVU and JNTUACEP for providing us feedback during our research which helped us to transit in right path towards our expected results. We are thankfull to our colleagues Nagaraju and Vishnu vardhan for continuous support through out the research period.

\section{REFERENCES}

Abbas, A., M.I. Khalil, S. Abdel-Hay and H.M. Fahmy, 2008. Expression and illumination invariant preprocessing technique for face recognition. Proceedings of the 2008 International Conference on Computer Engineering and Systems, November 25-27, 2008, IEEE, Cairo, Egypt, ISBN:978-1-4244-2115-2, pp: 59-64.
Agrwal, S.L., A. Yadav, U. Kumar and S.K. Gupta, 2016. Improved invisible watermarking technique using IWT-DCT. Proceedings of the 2016 5th International Conference on Reliability, Infocom Technologies and Optimization (Trends and Future Directions) (ICRITO), September 7-9, 2016, IEEE, Noida, India, ISBN:978-1-5090-1490-3, pp: 283-285.

Anonymous, 2019. Yale database: Computer science. Yale University, USA. https://cpsc.yale.edu/

Bhadu, A., R. Tokas and D.V. Kumar, 2012. Facial expression recognition using DCT, gabor and wavelet feature extraction techniques. Intl. J. Eng. Innovative Technol., 2: 92-95.

Chen, C. and J. Zhang, 2007. Wavelet energy entropy as a new feature extractor for face recognition. Proceedings of the 4th International Conference on Image and Graphics (ICIG 2007), Auguest 22-24, 2007, IEEE, Sichuan, China, ISBN:978-0-7695-2929-5, pp: 616-619.

Gupta, S.K., S. Agrwal, Y.K. Meena and N. Nain, 2011. A hybrid method of feature extraction for facial expression recognition. Proceedings of the 2011 7th International Conference on Signal Image Technology and Internet-Based Systems, November 28-Dec 1, 2011, IEEE, Dijon, France, ISBN:978-1-46730431-3, pp: 422-425.

Lajevardi, S.M. and M. Lech, 2008. Facial expression recognition using neural networks and log-gabor filters. Proceednigs of the 2008 International Coneference on Digital Image Computing: Techniques and Applications, December 1-3, 2008, IEEE, Canberra, Australia, ISBN: 978-0-7695-3456-5, pp: 77-83.

Liu, S.S., Y.T. Tian and D. Li, 2009. New research advances of facial expression recognition. Proceedings of the 2009 International Conference on Machine Learning and Cybernetics Vol. 2, July 12-15, 2009, EEE, Hebei, China, ISBN:978-1-4244-3702-3, pp: $1150-1155$.

Ou, J., X.B. Bai, Y. Pei, L. Ma and W. Liu, 2010 . Automatic facial expression recognition using Gabor filter and expression analysis. Proceedings of the 2010 2nd International Conference on Computer Modeling and Simulation Vol. 2, January 22-24, 2010 , EEE, Sanya, Hainan, China, ISBN:978-1-4244-5642-0, pp: 215218.

Ramadan, R.M. and R.F. Abdel-Kader, 2009. Face recognition using particle swarm optimization-based selected features. Intl. J. Signal Process. Image Process. Pattern Recognit., 2: 51-65. 
Ramasubramanian, D. and Y.V. Venkatesh, 2001. Encoding and recognition of faces based on the human visual model and DCT. Pattern Recognit., 34: 24472458.

Sellahewa, H. and S.A. Jassim, 2008. Illumination and expression invariant face recognition: Toward sample quality-based adaptive fusion. Proceedings of the 2008 IEEE 2nd International Conference on Biometrics: Theory, Applications and Systems, September 29-October 1, 2008, IEEE, Arlington, Virginia, USA., ISBN:978-1-4244-2729-1, pp: 1-6.

Shan, C., S. Gong and P.W. McOwan, 2009. Facial expression recognition based on local binary patterns: A comprehensive study. Image Vision Comput., 27: 803-816.
Tariq, U. and T.S. Huang, 2012. Features and fusion for expression recognition-A comparative analysis. Proceedings of the 2012 IEEE International Conference on Computer Society Computer Vision and Pattern Recognition Workshops, June 16-21, 2012, IEEE, Providence, Rhode Island, USA., ISBN:978-1-4673-1611-8, pp: 146-152.

Tyagi, S.K. and P. Khanna, 2012. Face recognition using discrete cosine transform and nearest neighbor discriminant analysis. Intl. J. Eng. Technol., 4: 311314.

Verma, D., V. Dhaka and S. Agrwal, 2013. An Improved average GABOR wavelet filter feature extraction technique for facial expression recognition. Intl. J. Innovations Eng. Technol., 2: 1058-2319. 